\title{
Quaderni
}

QUADERNI Communication, technologies, pouvoir

\section{Fabriquer l'action publique : une activité "politique" ?}

\section{Guillaume Gourgues}

\section{Q OpenEdition}

1 Journals

\section{Édition électronique}

URL : http://journals.openedition.org/quaderni/988

DOI : 10.4000/quaderni.988

ISSN : 2105-2956

\section{Éditeur}

Les éditions de la Maison des sciences de l'Homme

\section{Édition imprimée}

Date de publication : 5 mai 2016

Pagination : 123-129

\section{Référence électronique}

Guillaume Gourgues, « Fabriquer l'action publique : une activité "politique" ? », Quaderni [En ligne], 90 | Printemps 2016, mis en ligne le 05 mai 2016, consulté le 21 décembre 2020. URL : http://

journals.openedition.org/quaderni/988; DOI : https://doi.org/10.4000/quaderni.988 


\section{Lecture croisée}

\section{Fabriquer l'action publique : une activité "politique" ?}

par Guillaume Gourgues

Université de Franche-Comté

Laboratoires CRJFC/PACTE

La fabrique politique des politiques publiques. Une approche pragmatique de l'action publique. Philippe Zittoun

Paris, Presses de Sciences Po, 2013

1. On citera notamment J-G. Padioleau, L'État au concret, Paris, PUF, 1982 ; B. Jobert, P. Muller, L'État en action : politiques publiques et corporatismes, Paris, PUF, 1987. On pourrait également évoquer l'ouvrage de Gilles Massardier, qui s'apparente fortement à un essai, tout en se présentant dans un format de manuel : cf. G. Massardier, Politiques et actions publiques, Paris, Armand Collin, 2003.
Dans l'ouvrage issu de son habilitation à diriger les recherches récemment soutenue à Sciences Po Grenoble en 2012, Philippe Zittoun se livre à un exercice devenu rare dans la littérature française - pourtant pléthorique - en analyse des politiques publiques : la conduite d'un ouvrage, à visée théorique, écrit seul et récapitulant plusieurs travaux de recherche, n'étant ni un manuel, ni un ouvrage de vulgarisation. Il faut en effet remonter aux années 1980 pour trouver des démarches comparables ${ }^{1}$. Et autant le dire tout de suite : l'exercice est rondement mené, tant il ouvre une ligne théorique cohérente et des pistes de controverse fortes. La lecture que nous proposons de l'ouvrage suit ainsi ces deux aspects. On notera pour commencer les apports indéniables de l'approche de $\mathrm{Ph}$. Zittoun dans les débats traversant l'étude des politiques publiques, tout en nous interrogeant ensuite sur la principale hypothèse avancée par l'auteur : la dimension « politique » est centrale dans la fabrique des politiques publiques.

Pour décrire l'approche proposée par Ph. Zittoun, nous pourrions dire ceci : il s'agit de comprendre, à l'aide d'une approche pragmatique focalisée sur ce que font et disent les acteurs, comment ces derniers fabriquent l'action publique en s'accordant et s'affrontant par le biais du discours pour tenter de stabiliser (temporairement) un ordre social profondément instable. La dimension « politique » de ce processus tient précisément à cette intégration/ dissolution des facteurs de désordre dans un ordre apparent : produire des politiques publiques est une manière de garantir la cohésion d'une société, 
en traitant, même de manière insatisfaisante, les problèmes qui se posent. Cette dimension politique passe alors essentiellement par le discours : les arguments, contre-arguments, couplages (solutions/problèmes, solutions/ idéologie, solutions/instruments), récits apocalyptiques, auxquels se livrent sans cesse les acteurs les poussent à agir, alors même qu'ils peuvent aisément constater que leur action n'a que peu d'effets sur les problèmes.

L'opération discursive, trop souvent minorée par l'analyse des politiques publiques, est la trame qui permet de filer les opérations centrales de cette « fabrique politique » : l'émergence (chaotique) des problèmes (chapitre 1), la stabilisation des énoncés qui permet de coupler le problème à une solution (chapitre 2), la diffusion des énoncés par un jeu d'enrôlement (chapitre 3) et la prise de décision, reposant sur la « victoire » temporaire d'un énoncé sur ses concurrents (chapitre 4). Chacune de ces séquences, inspirées par l'inénarrable « grille séquentielle», est alors revisitée, retravaillée, et remise en cohérence, permettant d'ouvrir des pistes d'analyse tout à fait prolixes. Signalons au passage que l'auteur prend à revers une tendance générale à la «sacralisation » de la phase de mise en œuvre des politiques publiques, devenant le siège de toutes les (re)définitions des rapports de force et des évolutions de l'action publique ${ }^{2}$. En se limitant aux trois premières séquences classiques (émergence des problèmes, mise à l'agenda, décision), qu'il étend et redécoupe, $\mathrm{Ph}$. Zittoun propose des parcours de lecture (ou plutôt de relecture) des auteurs classiques de l'analyse des politiques publiques dont nous retiendrons deux apports majeurs.

Premièrement, l'ouvrage défend la cohérence d'une analyse « constructiviste » appliquée à l'action publique. L'auteur s'oppose à un usage trop restrictif, et pourtant largement répandu dans l'analyse des politiques publiques, du constructivisme, si ce paradigme reste privilégié dans certains secteurs de l'analyse (essentiellement l'étude des problèmes publics), les analystes ont rapidement tendance à se soustraire à la logique de construction sociale (qui ne concernerait que les acteurs), affirmant subitement détenir une connaissance objective des autres séquences (mise à l'agenda, décision). $\mathrm{Ph}$. Zittoun reprend en cela, sans toutefois s'y référer explicitement, le reproche fait par Dorothy Pawluch et Steve Woolgar ${ }^{3}$ au « charcutage ontologique » dont est souvent victime le constructivisme en sciences sociales. L'enjeu de la « décision », abordé frontalement dans l'ouvrage, est emblématique de ce problème. En effet, pour Ph. Zittoun, l'empressement de l'analyse

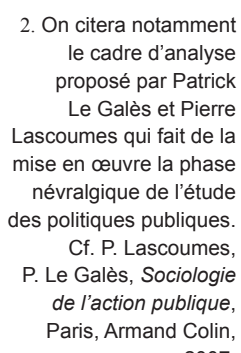

3. S. Woolgar D. Pawluch, 1985 « Ontological Gerrymandering. The anatomy of social problems explanations ", Social Problems, 32 (3), pp. 241-227. 
des politiques publiques à affirmer que la décision «n'existe pas », puisqu'elle supposerait des conditions sociales invraisemblables (unicité du décideur, de l'espace et du temps de décision), rend aveugle sur un enjeu majeur de la construction des politiques publiques, à savoir le rôle de la croyance des acteurs dans l'existence de la décision et « du » décideur. Ainsi, il est proposé de changer de posture : plutôt que de chercher à savoir si la décision « existe », il convient de comprendre comment chaque acteur établit une cartographie décisionnelle (qui décide, qui ne décide pas, qui peut influencer la décision), qui s'avère cruciale dans les rapports de force et la progression d'un énoncé de politiques publiques. Comme l'affirme Ph. Zittoun, « la question pour le chercheur n'est alors pas de savoir si cette cartographie des positions est vraie ou fausse, mais de comprendre que c'est la définition de cette carte à un temps t qui permet à l'acteur de sélectionner celui qu'il veut persuader » (p. 225). Le constructivisme constitue en cela l'épine dorsale de la proposition analytique de l'ouvrage, et lui permet de questionner efficacement les routines et les braconnages de l'analyse des politiques publiques.

Deuxièmement, l'ouvrage propose des déconstructions pédagogiques de certains passages incontournables et classiques de l'analyse des politiques publiques. Ainsi, on soulignera l'intérêt du chapitre 1 concernant l'émergence des problèmes, qui enrichit les outils de déconstruction des entreprises de problématisation du monde menées par les acteurs, regroupés en « opérations » distinctes (nommer un problème, créer un public de victimes, désigner des responsables, annoncer l'apocalypse et rendre nécessaire l'action). On relèvera également l'usage de la notion de « couplage » (chapitre 2) qui permet de comprendre comment problèmes et solutions se rencontrent aléatoirement et poussent les acteurs à justifier cette rencontre a posteriori, sans tomber dans la lecture simplificatrice d'un « référentiel global » qui se déclinerait dans tous les secteurs. L'analyse des coalitions qui sous-tendent la mobilisation des acteurs s'affrontant dans cette fameuse « fabrique » de l'action publique est également finement menée : ces dernières ne se fondent pas obligatoirement sur une concordance idéologique et intellectuelle absolue, mais engagent un travail permanent d'accord et de désaccord sur les frontières d'un énoncé collectif, aussi fragile que déterminant dans l'alliance des acteurs.

Mais l'apport principal de l'ouvrage, au-delà de ces indéniables points d'intérêt, constitue en réalité sa principale faiblesse. Cet apport tient à la sentence que $\mathrm{Ph}$. Zittoun n'a de cesse d'affirmer sans détour : toute politique 
publique relève bel et bien de l'activité politique. Mais qu'est-ce à dire exactement? La politique est ici ramenée à la production jamais achevée d'ordre et de désordre, revenant en cela aux définitions canoniques de l'objet d'étude de la science politique, cette fameuse « chance de faire triompher, au sein d'une relation sociale, sa propre volonté, même contre des résistances $»^{4}$. Suivant les mots de l'auteur : «montrer que la fabrique d'une politique publique constitue d'abord une activité politique revient à mettre en évidence que le travail définitionnel est une activité dont la spécificité est de permettre une dialectique antinomique entre l'ordre et le désordre politiques » (p. 20). Les fabriques de l'action publique participent donc du politique, puisqu'elles ordonnent temporairement le chaos social, fait de constructions problématiques, de débordements des normes et des règles, de remises en cause du système, des demandes élémentaires d'une population à satisfaire. Étudier l'action publique, c'est donc se poser « la » grande question de l'ordre politique et donc, en somme, faire de « la » science politique au-delà de tout découpage sous-disciplinaire abscons.

Cette volonté d'intégrer l'étude de l'action publique dans la science politique n'est pas une entreprise nouvelle ${ }^{5}$, mais conduit inexorablement $\mathrm{Ph}$. Zittoun à se situer à un niveau de discussion qui finit par poser problème. En effet, si Dewey, Lindblom, Dahl, Simon et même Foucault et Rancière sont invités à la table des discussions, parfois très longuement et souvent au risque de perdre le lecteur dans un océan de débats théoriques, les auteurs contemporains travaillant précisément sur le lien entre politique et action publique (le tandem infernal politics/policies) sont priés de rester sur la touche. On pourra d'ailleurs souligner un premier problème lié à cette stratégie d'écriture : la marginalisation de la dimension discursive dans les « travaux récents » nous semble quelque peu exagérée ${ }^{6}$. Mais le problème majeur qui se pose alors réside dans l'assise empirique de l'ouvrage, qui s'avère insatisfaisante aux vues de l'ambition théorique affichée (et réaffirmée en permanence) et laisse en suspens la question du positionnement de $\mathrm{Ph}$. Zittoun sur la place de la « variable politique » dans l'action publique. Pour le dire d'une formule, si $\mathrm{Ph}$. Zittoun s'emploie activement à montrer que les politiques publiques sont bien « gouvernées » (elles impliquent des luttes d'acteurs et ont des conséquences, en retour, sur les rapports de force), aucun élément n'est donné sur une autre question majeure : qui gouverne?

Or, c'est précisément à ce stade que l'ouvrage s'avère problématique. Le

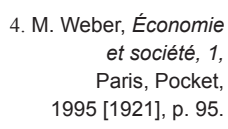

4. M. Weber, Économie et société, 1, Paris, Pocket, 1995 [1921], p. 95

5. B. Jobert, « L’État en action. L'apport des politiques publiques », Revue française de science politique, 35,4 , 1985, pp. 654-682.

6. Plusieurs travaux nous semblent en effet accorder une place non négligeable aux logiques discursives: c'est notamment le cas de Stephen Skoronek pour qui les types de leadership présidentiel se distinguent bien en fonction des capacités de « dialogue » et d'entente qu'ils permettent ou interdisent entre les acteurs clés du système politique ; dans sa lecture de la politique de la famille en Suisse, Daniel Kubler insiste lui aussi sur la centralité du travail argumentatif qui justifie le changement d'alliance entre libéraux, conservateurs et socio-démocrates. Cf. S. Skowronek, The Politics Presidents Make: Leadership from John Adams to George Bush, The Belknap Press of Harvard University Press, 1993 ; D. Kübler,

« Understanding the recent expansion of Swiss family policy. An idea-centred approach », Journal of Social Policy, vol. 36, n², 2007, pp. 217-237. 
7. A. Schafer, W. Streeck (dir.), Politics in the age of austerity, Polity Press, 2013.

8. N. Brenner, New state spaces: urban governance and the rescaling of statehood, Oxford, Oxford University Press, 2004.

9. D. Lorrain, «"La main discrète". La finance globale dans la ville", Revue française de science politique, vol. 6 , n6, 2011, pp. 10971122.

10. C'est notamment le cas en Italie, où le gouvernement est " confié » à des agents proches des marchés financiers, cf. C. Bouillaud, "L'Italie (2008-2013) : de la crise à cause de l'euro au miracle à venir grâce à l'euro ? ", Pôle Sud, vol. 39, n², 2013, pp. 11-34. On évoquera également l'extension des zones d'influence de la Banque Centrale Européenne à la faveur de la crise financière de 2008, cf. C. Fontan, « Frankenstein en Europe », Politique européenne, vol. 42, $n^{\circ} 4,2014$, pp. 22-45. hasard des calendriers de publication fait que d'autres ouvrages, parus au même moment, proposent une lecture radicalement divergente de la place du politique dans la production des politiques publiques : la «politique », au sens de la capacité des acteurs politiques (et parfois élus) à prendre des décisions en fonction d'enjeux politiques (comme les élections par exemple), aurait bel et bien disparu des facteurs explicatifs de l'évolution des politiques publiques ${ }^{7}$. L'austérité économique, la concurrence toujours plus forte entre autorités publiques, imposant un renouvellement des stratégies de développement nationales et urbaines ${ }^{8}$, réduiraient les marges de manœuvre « politiques » à néant, instaurant une voie toujours plus homogène et contrainte à tous les gouvernants du monde, devenant des entrepreneurs standardisés encadrés par des règles discrètes mais puissantes ${ }^{9}$ ou étant explicitement remplacés par des « techniciens » du sillon libéral ${ }^{10}$. L'actualité renforce, dans un tout autre domaine, l'idée d'un effacement apparent de la dimension politique dans la production de l'action publique : comment analyser, par exemple, la disparition quasi-totale de controverses parlementaires et partisanes lors de la mise en place de l'état d'urgence en France ? Sous cet angle, le titre de l'ouvrage - la fabrique « politique »- mérite donc d'être débattu.

En effet, plutôt que de s'engager sur cette piste de polémique importante, tant les conséquences pour la compréhension et l'équilibre de l'ensemble du système politique sont considérables, Ph. Zittoun multiplie les détours empiriques parcellaires qui, s'ils viennent illustrer son argumentation, ne relèvent pas de manière évidente du " politique ». On s'intéressera ainsi ponctuellement à des éléments empiriques disparates, issus des recherches antérieures de l'auteur, sur les ententes et mésententes entre acteurs concernant la politique du logement, la carte du bruit à Paris, les discours présidentiels sur la TVA sociale, sans pouvoir entrer dans une logique contrefactuelle qui « prouverait » que la politique y est bien présente. Mais ce problème apparaît surtout lors du dernier chapitre, intégralement consacré à une étude de cas (l'implantation d'un tramway à Paris), et qui joue un double rôle, à notre sens : montrer l'intérêt mais également, et surtout, les limites de l'approche proposée par $\mathrm{Ph}$. Zittoun.

L'étude du cas du tramway parisien permet ainsi de saisir la manière dont deux coalitions d'acteurs portent, depuis la fin des années 1980, deux tracés concurrents, tous deux appuyés sur des énoncés contraires et en lutte. Chacun enrôle des alliés, recompose ses arguments, gagne en légitimité et en force 
jusqu'à la victoire d'un des énoncés qui « remporte » l'adhésion du maire de Paris, dans un contexte manifestement marqué par un consensus politique facilement construit au-delà des clivages partisans. La linéarité de l'étude de cas permet de mobiliser la grille patiemment exposée dans l'ouvrage, mais ne cesse d'ouvrir une myriade de questions : au-delà du tracé, qui relève in fine d'un débat relativement « technique », y a-t-il un débat de fond sur l'opportunité du tramway « tout court » ? Comment se fait-il que Paris, à l'instar de très nombreuses villes françaises et étrangères, s'engage dans cette voie d'action publique ? Comment comprendre l'engouement partagé des villes pour le tramway, la rénovation des centres-villes, la défiscalisation compétitive, les projets « solidaires » dans les quartiers pauvres ? On peut alors inventorier quelques-uns des points aveugles de l'analyse : le rôle du lobbying des entreprises privées, a fortiori dans le secteur des transports urbains où règne une âpre concurrence entre un nombre réduit d'opérateurs (Kéolis et Transdev) ; la circulation des « bonnes recettes » de la « ville durable » dont on ne retiendra que les algorithmes les moins perturbants pour l'objectif prioritaire de la « compétitivité » urbaine et la dimension de marketing (le branding urbain) ; l'effacement des lignes conflictuelles, qu'elles soient partisanes ou qu'elles relèvent d'un débat public d'ensemble, au profit d'une technicisation toujours plus poussée des enjeux, destinées à établir un consensus entre élites à l'échelle urbaine, marginalisant ainsi l'intervention du « grand public ». De ce point de vue, l'évacuation de la mise en œuvre et des instruments qui la peuplent par l'approche défendue dans l'ouvrage, et donc d'une séquence où les acteurs entrent dans une justification a posteriori de décisions prises par d'autres ou sans projet politique clair, s'avère tout de même fondamentale et crée un certain déséquilibre analytique.

Ce décalage entre l'approfondissement de la cohérence interne de l'ouvrage et son manque de points de contact avec la littérature actuelle affaiblit l'intérêt des analyses proposées par Ph. Zittoun. Nichant le «politique» dans des débats dont on ne peut s'empêcher de mesurer la dimension technique, localisée et restreinte, l'auteur ne convainc pas du caractère inaltérable de la dimension politique de l'action publique. En effet, si l'on extrapole, on peut imaginer le maintien du cadre d'analyse de Ph. Zittoun dans un monde sans élections, sans élus, sans débat public, prenant la forme policée de la postdémocratie diagnostiquée par Colin Crouch ${ }^{11}$ : un monde de mise en débat d'alternatives techniques dans des cercles décisionnels plus ou moins ouverts et cooptés. On aimerait entendre l'auteur sur cette dimension aveugle de

11. C. Crouch, Post-Democratie, Zürich, Diaphanes, 2013. 
son cadre analytique, pour qu'il précise la portée «politique » (voire démocratique) de son propre modèle. On l'aimerait d'autant plus que la démocratie était bien l'horizon de réflexion de nombreux auteurs classiques dont $\mathrm{Ph}$. Zittoun prend plaisir à discuter les thèses. Ce thème sera, n'en doutons pas, au cœur des controverses soulevées par l'ouvrage qui n'en perd pas pour autant son statut d'essai analytique prolixe et passionnant. 
